\title{
MÉTACERCAIRE PROGÉNÉTIQUE CHEZ UN PLANORBE
}

\section{Par Robert Ph. DoLLFUS}

Depuis que j'ai introduit en biologie la notion de métacercaire progénétique, j'ai pu grouper plus d'une centaine d'observations de larves de distomes au stade metacercaria montrant, à des degrés divers, le phénomène de progénèse ; cependant, jusqu'à maintenant, aucun cas de progénèse n'était connu chez les métacercaires parasites de mollusques d'eau douce. Le cas que je présente aujourd'hui est donc le premier. Il a été découvert chez Planorbis planorbis (L.) des marais de Saint-Jean-de-Losne (Côte-d'Or), par P. Paris, qui m'a très aimablement communiqué la masse viscérale conservée en eau formolée, d'un planorbe où il l'avait observé (18-4-1932), ce dont je le remercie vivement. Cette masse viscérale renfermait: des sporocystes, des cercaires, de métacercaires enkystées progénétiques et des métacercaires libres, également progénétiques, avec œufs à maturité contenant un miracidium; il y avait en outre quelques jeunes métacercaires ne renfermant pas encore d'œufs et d'un peu plus petite taille (1).

Les sporocystes, cylindriques, s'atténuant aux extrémités, ont, en moyenne, une longueur de $0,75-0,80$ et un diamètre de $0,10-0,12$, ils renferment un nombre variable d'embryons de cercaires; dans un sporocyste j'en ai compté vingt-six, mais il y en a généralement beaucoup moins, parfois cinq ou six seulement.

La cercaire est leptocerque, le corps, en moyenne, est long d'environ 0,325 , large d'environ 0,125 ; la ventouse orale a un diamètre d'environ 0,060 , un peu inférieur à celui de l'acétabulum $(0,065)$; ce dernier a son centre légèrement en avant du milieu de la longueur du corps. La vessie est en Y, avec ses deux branches beaucoup plus courtes que la partie impaire; la bifurcation a lieu un peu en arrière de l'acetabulum et les branches se terminent sans atteindre tout à fait le bord postérieur de l'acetabulum. La coloration par la thionine fait apparaitre en rouge quelques territoires mal délimités

(1) Une de ces métacercaires ne renfermant pas encore d'œufs, était longue de 0,40 , large de 0,17 , avec une ventouse orale de 0,085 , un acetabulum de 0,085 environ, un pharynx de 0,025 , des ébauches testiculaires de 0,04 environ de diamètre. 
(en pointillé sur la figure 1). La queue, rétractée, ne mesure guère que 0,26 de long, mais dépasse 0,32 en extension, atteignant à peu près la longueur du corps; la base de la queue est insérée dans une encoche du bord postérieur du corps. Ne disposant que d'une optique des plus médiocres, je n'ai presque rien pu voir de l'organisa-

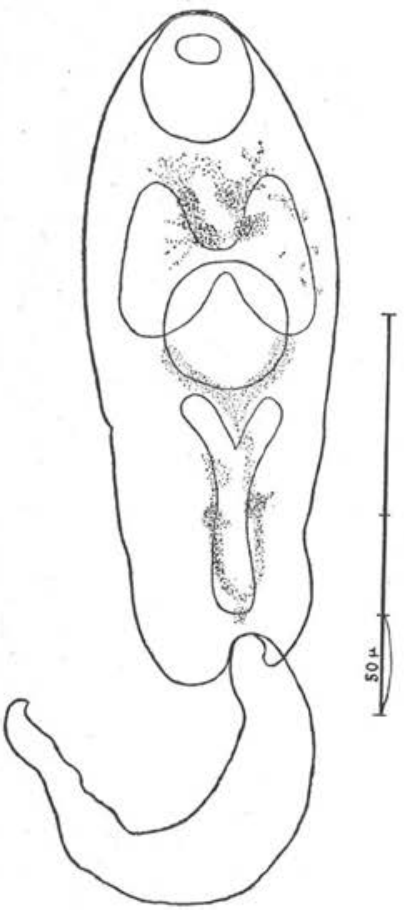

FIg. 1. - Cercaire de Planorbis planorbis (L.)

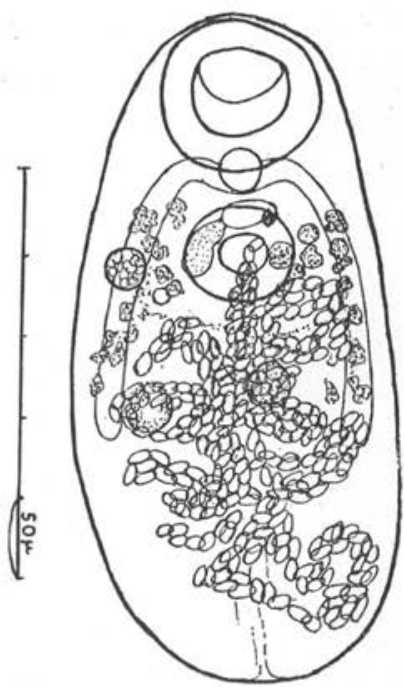

FIG. 2. - Métacercaire progénétique de Planorbis planorbis (L.) vue par la face ventrale.

tion de cette cercaire, néanmoins je ne crois pas qu'elle possède de glandes céphaliques et de stylet.

Les kystes métacercariens sont ellipsoïdaux $(0,59$ sur 0,36$)$ avec une paroi transparente extrêmement mince. Dans tous les kystes que j'ai examinés, la métacercaire était déjà bourrée d'œufs et ne différait pas des métacercaires non enkystées voisines dans les tissus de l'hôte et au même état de maturité sexuelle. Le corps est ovale et à peine moins épais que large (l'aplatissement ventral des individus bien étendus est faible). Les dimensions de trois indivi- 
dus étaient : 0,42 de long sur 0,20 de large, 0,525 sur $0,250,0,650$ sur 0,330 .

La ventouse orale est plus grande que l'acetabulum; pour les trois mêmes individus la ventouse orale mesurait respectivement : 0,10 , $0,14,0,175$ et l'acetabulum, respectivement : $0,065,0,10,0,14$. L'acetabulum est peu profond et son bord postérieur est généralement situé au milieu de la longueur du corps ou un peu en avant; mais

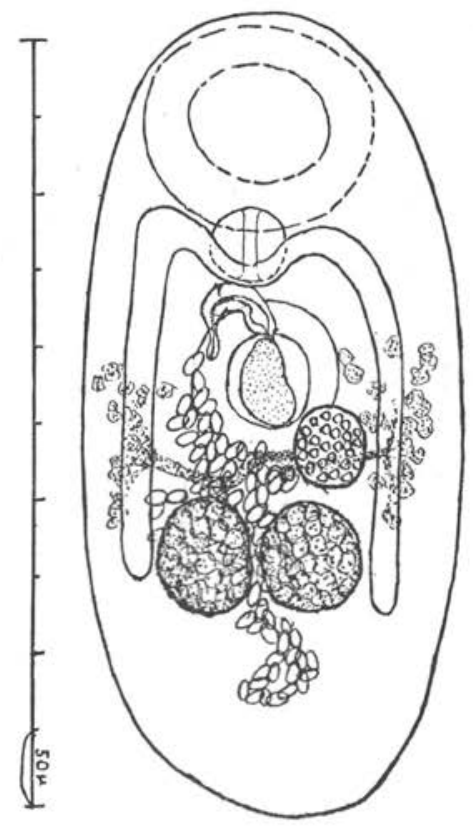

FIg. 3. - Métacercaire progénétique de Planorbis planorbis (L.). Autre exemplaire vu par la face dorsale

chez les individus dont la région antérieure du corps est un peu rétractée, le bord postériẹr de l'acetabulum peut se trouver plus ou moins en arrière de la mi-longueur du corps.

La cuticule est très finement striée transversalement mais il n'y a aucune trace de spinules.

Le prépharynx est nul, de même l'œsophage. La bifurcation a lieu immédiatement au bord postérieur du pharynx, qui est sphérique, avec un diamètre d'environ 0,026 pour les petits individus, 0,050 pour les moyens, 0,065 pour les grands. Les branches de la bifurcation se dirigent d'abord en dehors et en avant, vers la face 
dorsale, puis se recourbent en direction postérieure, pour se terminer plus ou moins loin dans le troisième quart de la longueur du corps, en gardant sur tout leur parcours le même diamètre (18 à $25 \mu$ selon les individus).

Les deux testicules sont globuleux, à bords entiers, leur diamètre varie selon les individus de 40 à $70 \mu$ environ, ils sont tous les deux au même niveau (ou à peu près), parfois très rapprochés l'un de l'autre, parfois éloignés; entre eux passe la portion impaire de la vessie. L'ovaire, sphérique, d'un diamètre de 25 à $70 \mu$ est situé plus ou moins loin en avant du testicule droit et au contact de la paroi dorsale du corps, son centre est à un niveau variant entre celui du centre de l'acetabulum et celui du bord postérieur de l'acetabulum. Un peu à gauche de l'ovaire et au niveau du bord inférieur de celui-ci, se trouve la glande de Mehlis, plus ou moins volumineuse. Les vitellogènes sont représentés, de chaque côté, par un petit groupe de follicules disposés le long des cæca sur une hauteur variable, leur niveau antérieur pouvant dépasser un peu celui du bord antérieur de l'acetabulum, leur niveau postérieur pouvant atteindre celui du bord postérieur des testicules. Vers le niveau de la moitié antérieure de l'acetabulum, les vitellogènes droit et gauche se rapprochent parfois beaucoup mais sans venir au contact l'un de l'autre. Le vitelloducte transverse, assez gros, s'étend d'un cæcum intestinal à l'autre, un peu en arrière du bord postérieur de l'acetabulum. Je n'ai pas vu de receptaculum seminis; s'il en existe, il est très petit. L'utérus décrit de nombreuses sinuosités descendantes et ascendantes, en avant et en arrière des testicules, arrivant même à remplir, chez certains individus, presque tout l'espace situé entre les organes, dans toute la partie du corps postérieure au niveau du centre de l'acetabulum, sans toutefois dépasser latéralement les cæca intestinaux. Les œufs, brunâtres, operculés, mesurent environ $38 \mu 5$ sur 19 , à maturité ; cependant, on trouve aussi des ceufs un peu plus petits $(33,5 \times 18,34 \times 18,36 \times 19)$ qui contiennent un embryon en voie de développement ou même un miracidium paraissant presque achevé.

L'appareil séminal mâle comprend une poche du cirre relativement petite, renfermant un cirre très petit et des cellules prostatiques. La poche du cirre est située au contact de la paroi antérodorsale de l'acetabulum et s'ouvre un peu à gauche de la ligne médiane sur le bord même de l'acetabulum, par une petite papille conique. Dorsalement à l'acetabulum et aboutissant à l'extrémité distale de la poche du cirre, se trouve une vésicule séminale 
externe sacciforme, relativement grosse, qui, ainsi que je l'ai constaté sur des coupes, est bourrée de spermatozoïdes.

De l'appareil excréteur, je n'ai vu que la vessie, en Y ; le tronc médian passe entre les testicules et se bifurque immédiatement
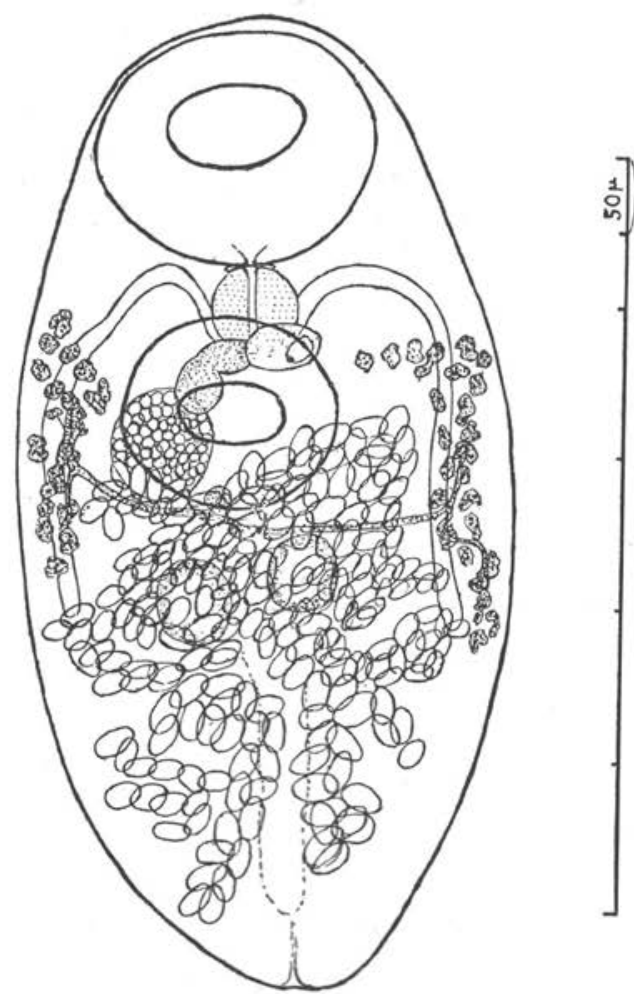

FIG. 4. - Métacercaire progénétique de Planorbis planorbis (L.). Autre exemplaire vu par la face ventrale

après, chaque testicule restant au contact de la branche correspondante; les deux branches sont courtes, n'atteignant pas, ou à peine, le niveau du bord postérieur de l'acetabulum.

Cette métacercaire appartient évidemment aux Lepodermatoidea (1). On retrouve une disposition semblable des testicules au

(1) J'ai recherché si, parmi les nombreuses larves de distomes parasites de planorbes et autres gastéropodes d'eau douce, décrites jusqu'à présent, il n'y en avait pas une à laquelle l'on pouvait rapporter celle trouvée par P. Paris ; je n'ai trouvé aucune description qui corresponde. 
même niveau, ou presque, chez quelques Lepoderma, par exemple L. cloacicola (Lühe), chez des Astiotrema, chez des Renifer, chez Leptophallus, etc... Toutefois, la métacercaire progénétique de planorbe ne semble correspondre exactement à aucun des genres bien bien caractérisés de Lepodermatidæ et Reniferidæ. Comme il y a une volumineuse vésicule séminale non comprise (à moins d'erreur d'observation de ma part) dans la poche du cirre, j'avais pensé plus particulièrement à Leptophallus ; la brièveté des cæca intestinaux, l'extension de l'utérus, les dimensions des œufs, me paraissaient compatibles avec ce genre, mais chez la seule espèce du genre : L. nigrovenosus (Bellingh.) $=L$. signatus $($ Duj.), la cuticule est spinulée, alors qu'elle est nue chez notre métacercaire; de plus, la bifurcation de la vessie a lieu entre les bords postérieurs des testicules, alors que, chez notre métacercaire, elle semble bien avoir lieu un peu plus en avant.

A mon avis, l'adulte auquel correspond la métacercaire progénétique du planorbe, peut très bien présenter avec celle-ci quelques différences morphologiques et l'on risquerait, en assignant un genre à la métacercaire, que ce genre ne soit pas celui auquel appartient réellement l'espèce. Il s'agit d'une espèce dont le cycle évolutif comporte ou non, selon les circonstances, une forme adulte dans le vertébré hôte définitif ; dans le cas présent, nous ne connaissons pas le cycle dit « normal », mais seulement le cycle progénétique; il faut attendre, pour une identification précise, le résultat de recherches dans la nature et d'infestations expérimentales.

\section{RÉSUMÉ}

Nous signalons le premier cas connu d'une métacercaire progénétique chez un mollusque d'eau douce. Le même planorbe héberge les sporocystes, les cercaires, les métacercaires enkystées et des métacercaires libres, vraisemblablement sorties de leur kyste.

Ces métacercaires atteignent la maturité sexuelle même dans leur kyste, elles produisent des œufs en grand nombre, ces œufs contiennent un embryon en voie de développement. La présence de spermatozoïdes dans la vésicule séminale permet d'admettre : qu'il n’y a pas développement parthénogénétique, mais fécondation (autofécondation lorsque la métacercaire est incluse dans son kyste), que le miracidium en formation n'est pas constitué seulement par des tissus somatiques. 
L'on se trouve en présence d'une espèce pouvant présenter, très probablement, deux cycles évolutifs : l'un abrégé, se passant tout entier dans le premier hôte, l'autre complet, que nous ne connaissons pas, comportant un adulte dans un vertébré hôte définitif.

Comme il y a vraisemblablement des différences morphologiques entre la métacercaire progénétique dans le planorbe et l'adulte de même espèce parasite dans le vertébré hôte définitif, nous estimons qu'il est préférable d'attendre de connaître cet adulte pour préciser à quel genre de Lepodermatoidea appartient notre espèce.

\section{Bibliographie}

Dollfus (R.-Ph.). - Polyxénie et progénèse de la larve métacereaire de Pleurogenes medians (Olsson). C. R. Acad. Sc., CLXXIX, 1924, p. 305-308, 1 fig.

- Sur une métacercaire progénétique d'hémiuride (Trémat-Digen). Bull. Biol. France et Belgique, LXI, 1927, p. 49-58, fig. 1-3.

- Existe-t-il des cycles évolutifs abrégés chez les Trématodes digénétiques? Le cas de Ratzia parva (Stossich, 1904). Ann. parasitol., VII, 1929, p. 196-203, fig. 1-5.

Joyeux (Ch.), Noyer (Rondeau du) et BaEr (J.-G.). - L'activité génitale des métacereaires progénétiques. Bull. soc. pathol. exotique, XXIII, 1930, p. $967-977$.

Mathias (P.) - Contribution à l'étude du cycle évolutif d'un trématode de la famille des Pleurogenetinæ Lss. (Pleurogenes medians Olss.). Bull. soc. zool. de France, XLIX, 1924, p. 375-377, fig. 1-4. 